\title{
Tumores Ováricos Malignos de las Células Germinales en la Infancia y Adolescencia, Experiencia de 14 Años
}

\author{
Dra. Mónica Muñoz O. ${ }^{1}$; Dra. M. Inés Sins D. ${ }^{2}$; Dr. Juan José Latorre L. ${ }^{3}$; \\ Dra. Victoria Beresi R. ${ }^{4}$; Dr. Juan Quintana B. ${ }^{4}$ Dr. Humberto del Pozo P. 5
}

\section{Malignant Gern Cell Ovarian Tumors in Infancy and Adolescent}

In a fouurteen years period (1971-1985) 821 patients with solid tumors were seen at a pedjatric oncology service in Santiago: 18 patients had malignant germ cell tumors of the ovary. Their age at first consulation was 10 months to 13 years (median 9 years). There were 9 case $(50 \%)$ of inmature teratomas, 4 (22\%) of endodcrmal sinus tumors, 3 (17\%) of embryonal carcinoma and 2 (11\%) of dysgerminoma. The most important prognostic factor was the presence and extent of tumor spread beyond the ovary, as expressed by the currently used cinical staiging (F.I.G.O.). All patients were treated by surgery, plus post-opecative chemotherapy in $16 / 18$ and additional irradiation in $5 / 18$. All patients in stages [ and $\mathbf{~ I l ~ h a v e ~ s u r v i v e d ~ f r o m ~} 37$ to 105 months; $6 / 9$ girls with stage III tumors have survived by. 2 to 113 months: $3 / 9$ died after follow-up of 4.5 and 10 months each. Only $2 / 6$ patients with stage IV tumors are actually surviveing ( 20 and 93 months of follow-up); $4 / 6$ died after one to ten months. Survival in cases of inmature tera1ona was $6 / 9$, in endodermal sinus tumor was $2 / 4$, in embryonal carcinoma $1 / 3$ and in dysgerminoma $2 / 2$. Post-operative gynecologic evaluation detected metastatis in two patients and recurrence in another two girls.

iKey words: Tumors. Ovarian. Malignant. Girls. Malignant teratoinas. Dysgeminoma. Embryonal carcinoma. Endodermal sinus tumors).

En la niñez la mayoría de los tumores ováricos derivan de las células germinales (más del $60 \%$ ) $^{3}$ a diferencia de lo que ocurre en la mujer adulta (menos del 30\%). Los teratomas maduros son los tumores ováricos derivados de células germinales ${ }^{3}$ más frecuentes (más de $58 \%$ ).

La medición de substancias relacionadas con tumor, para el diagnóstico y seguimiento de pacientes con procesos malignos, detección de metástasis y recidivas, juega un rol cada vez más importante en la clínica oncológica ${ }^{11}$. Con el advenimiento de radioinmunoensayo sensible y específico, se ha encontrado que las concentraciones de alfatetoproteína, gonadotropina coriónica (HCG) o ambas están aumentadas en pacientes con tumores testiculares $y$ ováricos malignos de las células germinales.

El objetivo de este estudio fue describir la frecuencia con que ocurrieron los diferentes tipos histológicos de los tumores ováricos de las células germinales registrados en un Centro oncológico de Santiago en 14 años, sus características

1. Depto, Ginecología Infantil y Adolescencia.

2. Jefe Depto: Ginecología Infantil y Adolescencia.

3. Jefe Depto. Anatomía Patológica.

4. Servicio Oncología.

5. Jefe Servicio Oncología Hospital Dr. Luis Calvo Mackenna. clínicas evolutivas y la respuesta al tratamiento según su tipo histológico y la etapa clínica en que los pacientes consultaron por primera vez.

\section{MATERIAL Y METODO}

Se analizaron los antecedentes de 18 pacientes que fueron atendidos y registrados en el Centro Oncológico del Hospital Luis Calvo Mackenna en los 14 ar̂os transcurridos entre los meses agosto de 1971 y 1985 , que tenian tumores ováricos malignos de las células germinales, ordenadas según la clesificación histológica de la Organización Mundial de la Salud (Apéndice 1), El estadio clínico de cada paciente fue clasificado de acuerdo con los criterios adoptados por la Federación Internacional de Ginecología y Obstetricia (F.J.G.O.), en 1974 (Apéndice 2). El análisis histológico de las muestras obtenidas por biopsia y los estudios citológicos de líquido peritoneal fueron hechos por médicos del Departamento de Anatomía Patológica del Hospital. La concentración sérica de alfafetoproteína se midió mediante radioinmunoensayo.

\section{RESULTADOS}

Entre 821 tumores sólidos registrados en el periodo de estudio eran tumores ováricos $(4,11 \%)$ y de éstos $18(53 \%)$ eran de naturaleza maligna.

Los tumores malignos identificados fueron: 9 teratomas inmaduros ( $50 \%), 4$ tumores del seno endodérmico $(22 \%), 3$ carcinomas embrionarios (17\%) y 2 disgerminomas (11\%). 
La edad de presentación de ellos fluctuó entre los 10 meses y 13 años, con una mediana de 9 años, acumulándose la mayoría de las pacientes entre los 10 y 14 años (11/18) (Figura 1).

El motivo más frecuente de consulta fue aumento de volumen abdominal, con masa palpable, en el $60 \%$ de los casos, que era dolorosa en la mitad de ellos. Tres pacientes ingresaron por abdomen agudo (17\%). Los hallazgos más destacados en la anamnesis y el examen físico de ingreso se enumeran en la Tabla 1.

Entre la primera consulta y el diagnóstico definitivo transcurrieron entre 2 días y 9 meses, con una mediana de 10 dias; el examen ginecológico adecuado se hizo únicamente en 2 pacientes y sólo en 7 (39\%) se hizo correctamente el diagnóstico preoperatorio de tumor ovárico, en otras 3 se pensó en tumores abdominales, en 2 otros tumores, abdomen agudo en 3 , embarazo en una, y no se formularon hipótesis en 2 pacientes.

\section{Tabla 1}

Síntomas y Signos más Destacados en la Primera Consulta de 18 Pacientes con Tumores Ováricos dc las Células Germìales

\begin{tabular}{lrr}
\hline & $\mathrm{N}$ & $\%$ \\
\hline Aumento de volumen & 11 & 61.0 \\
Masa palpable & 11 & 61.0 \\
Dolor abdominal & 6 & 33.0 \\
Circujación colateral & 5 & 28.0 \\
Fiebre & 3 & 17.0 \\
Baja de peso & 3 & 17.0 \\
Edema extremidades & 2 & 11.0 \\
Vómitos & 2 & 11.0 \\
Anorexia & 1 & 5.6 \\
Taquipnea & 1 & 5.6 \\
Dificultad respiratoria & 1 & 5.6 \\
\hline
\end{tabular}

El reexamen de los especímenes en el centro oncológico condujo a algún tipo de modificación de los diagnósticos del centro de referencia en 4/14 (28,5\%) de los casos. La forma histológica predominante fue el teratoma inmaduro. Con las excepciones de una paciente con carcinoma embrionario, que consultó en etapa I, y otras 2 con disgerminoma y tumor del seno endodérmico que to hicieron en etapa II, 11/18 niñas (83\%) consultaron tardiamente en estadios III y IV de enfermedad ( $F$ igura 2)

Todas las niñas con teratomas inmaduros y el $75 \%$ de las portadoras de tumor del seno endo. dérmico y carcinoma embrionario consultaron tardíamente en etapa III y IV (Figura 2).

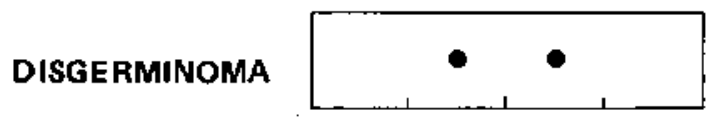

TUMOR DEL SENO ENDODERMICO

\section{CARCINOMA EMBRIONARIO}

\section{TERATOMA INMADURO}

\section{TIPO HISTOLOGICO (O.M.S.)/ESTADIO CLINICO (F.I.G.O.) AL MOMENTO DEL DIAGNOSTICO.}

Figurn 2: Etaps clínica en el momento del diagnóstico según tipo histológico de las lesiones en 18 niñas con tumores ováricos malignos.

El tratamiento fue quirúrgico en todas las pacientes, 16 de ellas $(89 \%)$ recibieron además quimioterapia y 5 cobaltoterapia $(28 \%)$. La

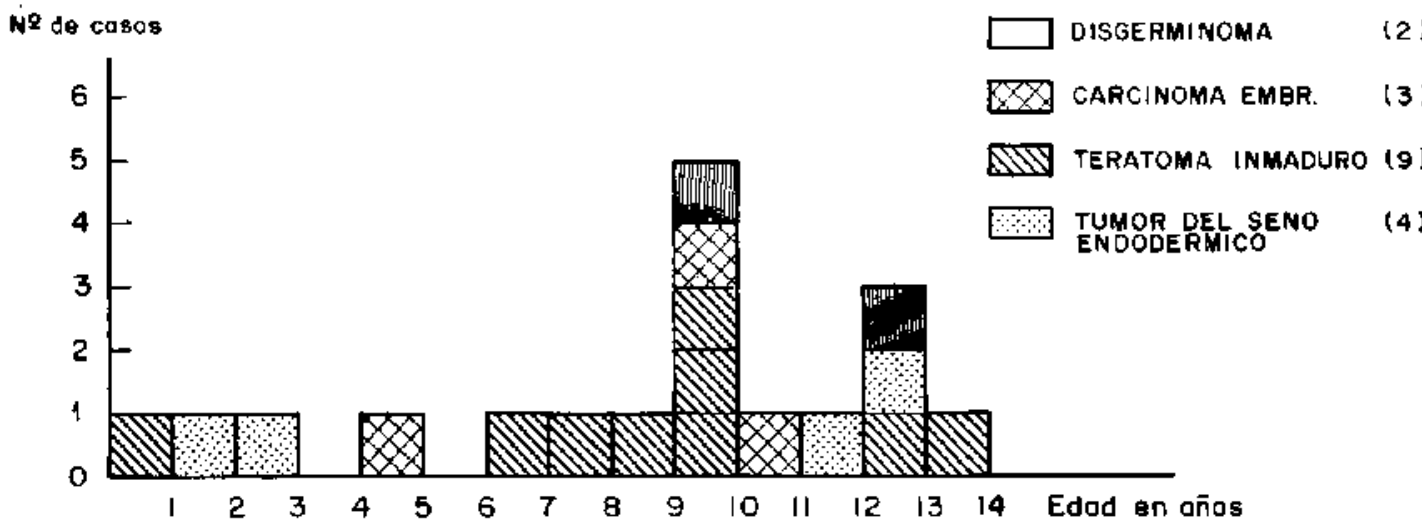

Figura 1: Distribución por edad de presentación y tipo histológico en 18 niñas con tumores ováricos malignos. 
quimioterapia fue hecha con vincristina, actinomicina D y ciclofosfamida (V.A.C.) en 7 pacientes; otras 5 nifas recibieron esas mismas drogas y además adriamicina (V.A.C.A.): en otros 3 casos la quimioterapia combinada se realizó con vinblastina, bleomicina y isplatinum (V.B.P.) y en uno con metotrexato, actinomicina D y ciclofosfamida (M.A.C.).

Todas las pacientes (3/3) en estadio I $y$ II (F.I.G.O.) están vivas al cabo de 37,68 y 105 meses; $6 / 9$ en estadio IIl han sobrevivido entre 2 y 113 meses, las otras fallecieron a $\operatorname{los} 4,5$ y 10 meses; $2 / 6$ pacientes en estadio IV viven 1 y 93 meses, las otras 4 fallecieron entre el primero $y$ décimo mes. En total hay 11 sobrevivientes (61\%) hasta el momento, en 2 se han detectado recidivas del tumor, en otras 2 , masas tumorales correspondientes a metástasis abdominales. En las siete restantes los exámenes ginecológicos y de otra indole no han demostrado alteraciones posteriores (Figura $3 \mathrm{a} \mathrm{y}$ b).

La A.F.P. se pudo medir en $15 / 18$ niñas ( $83 \%$ ) y estaba elevada en $8 / 15 \operatorname{cosos}(53 \%)$ : $4 / 8$ pacientes con teratoma inmaduro, $3 / 4$ con tumor de seno endodérmico y en la única nifia con carcinoma embrionario, dando resultados negativos en todos los casos con disgerminoma (2/2). Los casos negativos de A.F.P.

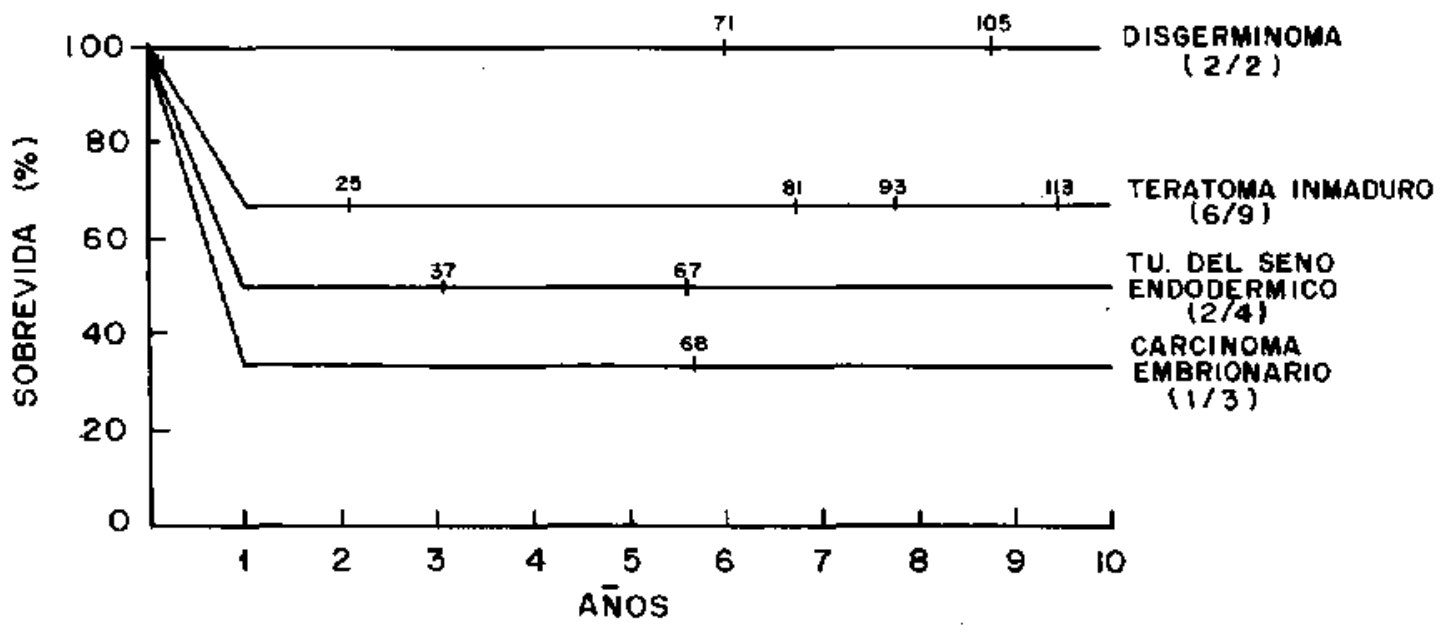

SOBREVIOA (\%) EN EL TIEMPO (AÑOS) / TIPO HISTOLOGICA (O.M.S.)

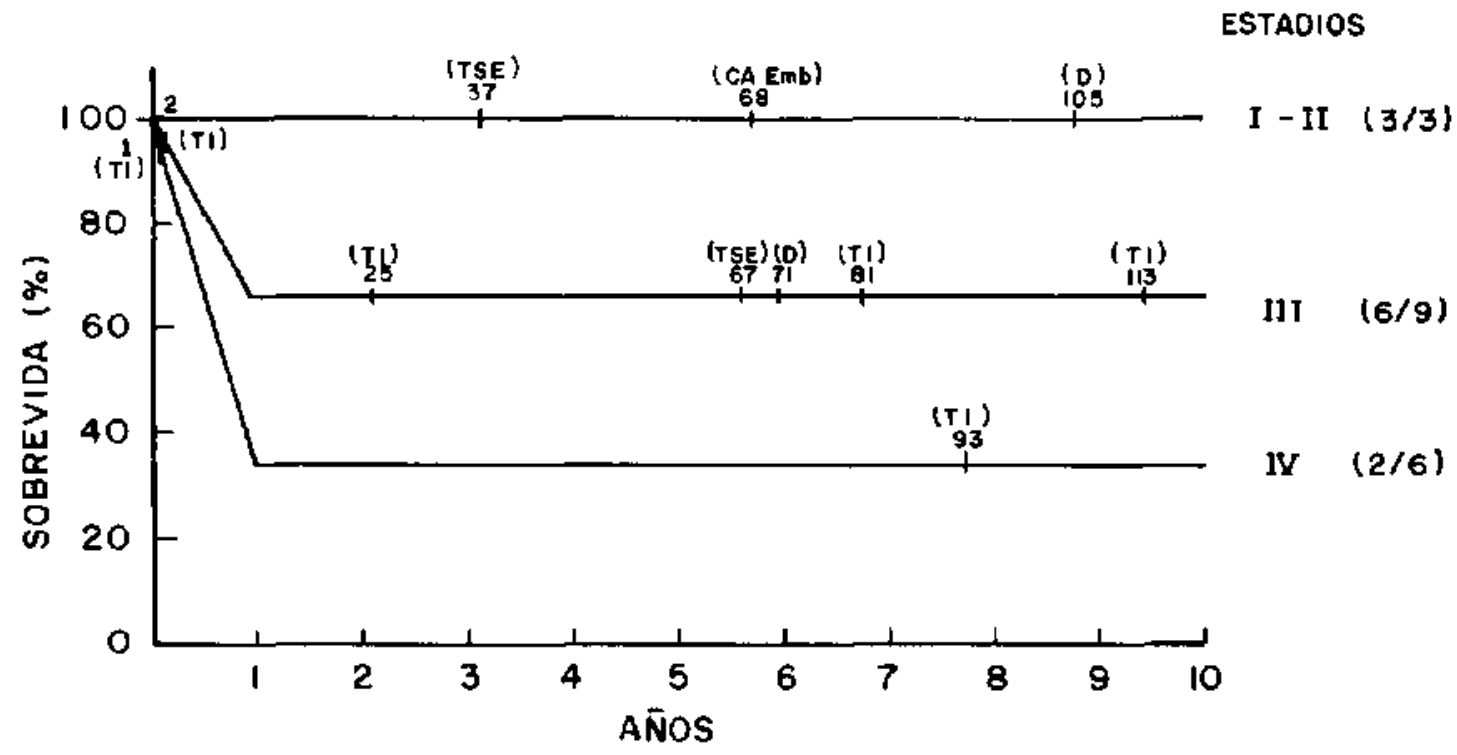

SOBREVIDA (\%) EN EL TIEMPO (AÑOS)/ ESTADIO CLINICO (F.I.G.O.)

Figura 3: Sobrevida ( $\%$ en años de las pacientes con tumores ováricos malignos.

a) según tipo histológíco; b) según estado clínico. 
en teratomas inmaduros y tumor del seno endodérmico corresponden a niñas en que la proteína se investigó tardíamente, por lo general un mes después de la intervención quirúrgica.

Sólo en 3/15 niñas con A.F.P. positiva previa se pudo medir ésta después del tratamiento quirúrgico y de comenzar la quimioterapia; observándose su desaparición en una niña con teratoma inmaduro un mes después y en otras dos con tumores del seno endodérmico, al segundo y décimo cuarto mes de seguimiento.

\section{COMENTARIO}

Aunque los tumores ováricos en las niñas son poco frecuentes ${ }^{1}$, constituyeron en esta serie $4,1 \%$ del total de los tumores sólidos registrados en un servicio de oncología, el $97 \%$ de los tumores genitales en niinas. Otros auto. res han encontrado incidencias de tumores genitales, hasta la edad de 14 años, de $4,9 \%$, siendo también el ovario el sitio más común. mente afectado $(3,3 \%)^{2}$.

Los tumores ováricos en general son raros antes de los 6 años de edad ${ }^{1}$ y su incidencia aumenta con la edad: en 17 series se encontró que la edad de presentación variaba entre recién nacidos y 4 años en $17 \%$ de los casos; $28 \%$ tenían entre 5 y 9 años y un $55 \%$ desde 10 hasta 14 años $^{3}$. En nuestra experiencia $11 / 18$ tumores $(20 \%)$ aparecieron en niñas menores de 6 años y el $22 \%$ de ellos eran neoplasias malignas.

La frecuencia relativa de los diferentes tipos histológicos de los tumores ováricos en niñas es inversa a la encontrada en la mujer adulta. En niñas y adolescentes predominan los tumores ováricos de las células germinales ${ }^{1}$. A medida que avanza la edad los tumores del estro. ma đel cordón sexual y de origen epitelial aumentan en frecuencia particularmente después de la meñarquia.. Así entre los 15 y 17 años los tumores epiteliales constituyen casi un tercio de los tumores ováricos y a medida que la edad avanza, aumentan los tumores serosos, mucinosos, endometroides, de tal forma que en conjunto los tumores epiteliales comunes constituyen más del $70 \%$ de los tumores ováricos en la mujer adulta.

Los tumores ováricos malignos en la niñez son comúnmente grandes, la mayoría mide más de $10 \mathrm{~cm}$ de diámetro cuando se detec. tan. En la actual revisión, a pesar del tiempo relativamente corto transcurrido entre la primera consulta y el diagnóstico, la gran mayoria, consultó en estadios tardios de enfermedad. Los estadios de cáncer ovárico se refieren a los hallazgos clínicos, quirúrgicos e histológicos. El estadjo o grado describe la extensión de la enfermedad en la paciente y es útil para planificar el tratamiento, formular el pronós. tico y comparar diferentes protocolos de tratamiento en distintas instituciones ${ }^{2}$.

Entre los tumores ováricos malignos de las células germinales, se señala que el más frecuente es el disgerminoma, siguiendo en orden de frecuencia, el tumor del seno endodérmico, el teratoma inmaduro, el carcinoma embrionario y el coriocarcinoma ${ }^{1}$. En esta revisión el disgerminoma fue el menos frecuente y se caracterizó por su tendencia a la diseminación linfática paraaórtica y su extrema radiosensibilidad, siendo el mas frecuente el teratoma inmaduro de crecimiento muy rápido. La histogénesis de los tumores de las células germinales ha causado debate $y$ confusión por muchos años. La teoría más aceptada sugiere un origen en la célula germinal primordial ${ }^{4}$. La presencia de elementos tumorales de histologia diferente dentro del mismo tumor es la base que apoya una histogénesis común. La migración de las células germinales en el embrión del saco vitelino a la gónada ${ }^{5}$ determina que los sitios más comunes en que se ubican estos tumores sean el retro-peritoneo, la pared abdominal posterior, el área sacrococcigea y las gónadas. Lugares menos comunes, que pueden resultar de migración aberrante, de pocos milímetros, de las células germinales, en etapas tempranas del desarrollo del embrión, son el mediastino, la hipófisis, la faringe, el riñón y el tracto genital.

El tratamiento del cáncer ovárico dependió de la extensión del tumor en el momento de la intervención quirúrgica y del tipo celular. Lo más importante es que el diagnóstico histológico sea correcto: la quimioterapia combjnada usada fue la descrita por diversos autores en. la literatura ${ }^{6}, 7,8,9$. Una combinación racional de drogas antineoplásicas es aquella en que cada agente antineoplásico actúa por separado de los otros sobre el tumor, con diferentes toxicidad y mecanismo de acción pero con sinergismo clínt. co. La combinación de V.B.P. cumple con estas condiciones ${ }^{10}$.

La radioterapia se utilizó en disgerminoma y fue acompañada de quimioterapia combinada en estadios tardíos de teratoma inmaduro, con sobrevida de todos estos casos.

Es importante la evaluación ginecológica pre y post operatoria. En nuestra revisión no hubo falsos positivos y el seguimiento post operatorio permitió detectar oportunamente recaidas.

La importancia que tiene la medición de A.F.P. en pacientes con tumores ováricos en el 
diagnóstico y seguimiento de ellos queda ampliamente ilustrada en esta experiencia.

En el futuro nos proponemos estudiar el cariotipo de las pacientes que sufran este tipo de tumores con el fin de relacionarlo con el comportamiento clínico y grado histológico, pues se ha descrito que los tumores de grados histológicos 0 , 1 y 2 en pacientes con cariotipos femeninos normales $(46 \mathrm{XX})$ tienen mejor pronóstico ${ }^{12}$.

\section{RESUMEN}

18 tumores malignos del ovario fueron encontrados entre 821 tumores sólidos registrados en un centro oncológico pediátrico de Santiago en un periodo de 14 años, entre 1971 y 1985 . La edad de presentación de los tumores varió entre 10 meses y 13 años (mediana 9 años), 9 casos correspondiecon a teratomas inmaduros ( $50 \%)$, a 4 tumores del seno endodérmico, 3 a carcinomas embrionarios y 2 a disgerminomas. El factor pronóstico más importante fue la presencia y extensión del tumor más allá del ovario: de acuerdo con las etapas clinicas en uso (F.I.G.O.). El paciente consultó en etapa I, 2 lo hicieron en etapa II, 9 en etapa IIJ y 6 en etapa IV. todos fueron tratados con cirugía, $16 / 18$ con quimioterapia y $5 / 18$ con radioterapia además de los procedimientos anteriores. Todas las pacientes en etapas I y II están vivas después de 37 y 105 meses de seguimiento; 6 a 9 niñas en etapa III han sobrevivido entre 3 y 113 meses, las otras 3 fallecieron 4, 5 y 10 meses después de su admisión. 4 de 6 pacientes en etapa IV fallecieron entre el primero y décimo mes de seguimiento. 2 han sobrevivido.1 y 93 meses respectivamente.

Entre 11 sobrevivientes 2 tienen metastasis abdominal y en 2 ha recidivado el tumor.

\section{Apéndice 1}

Clasificación Histológica de Tumores Ováricos*

\section{A. Tumores Epiteliales Comunes}
1. Seroso
2. Mucinoso
3. Endometrioide
4. De células claras (mesonefroide)
5. De Brenner
6. Mixto
7. Indiferenciado
8. No clasifjicados

B. Tumores del Estromn de Cordones Sexuales

1. Tumores de células de granulosa y de estroma:

a) Tumór de células granulosa

b) Grupo de tecoma - fibroma

1. Tecoma

2. Fibroma
2. Androblastomas: tumores de cćlulas de SertoliLeydig

3. Ginandroblastomas

4. No clasificados.

C. Tumores de Células Lipoides

D. Gonadoblastomas

E. Tumores de Células Germinativas

1. Disgerminoma

2. Tumor de seno endodérmico

3. Carcinoma embrionario

4. Poliembrioma

5. Coriocarcinoma

6. Teralomas
a) Teratoma inmaduro
b) Teratoma maduro
c) Tumores monodérmicos y especializados

7. Formas mixtas

\section{F. Trastornos "Canceriformes" \\ G. Tumores Metastásicos Secundarios}

* Organización Mundial de la Salud (OMS)

Apéndice 2.

Estadio Clínico de los Tumores Ováricos Matignos*

\section{Etapa 1}

a) Tumor limitado a un ovario - sin ascitis

b) Tumor limitado a ambos ovarios - sin ascitis

c) Tumor limitado a uno o ambos ovarios pero con ascitis o lavado peritoneal $(t)$

\section{Etapa II}

a) Tumor ovárico con extensión a útero y/o trompas

b) Con extensión a otros tejidos pelvianos

c) Etapa IIa ó IIb, pero con ascitis o lavado peritoneal $(+)$

\section{Etapa III}

a) Tumor de uno a ambos ovarios con metástasis intrabdominal

\section{Etapa IV}

4) Tumor de uno o ambos ovarios con metástasis a distanca fuera de la cavidad abdominat (pulmón, cerebro).

NOT A: Si existe derrame pleural debe existir citología (+) en el líquido pleural para poder catalogarlo en Etapa JV.

Metástasis intrahepática automáticamente clasifican al tumor en etapa IV.

* Federación Internacional de Gineoclogía y Obstetricia (F.I.G.O.).

\section{REFERENCIAS}

1. Larry J. Copeland: Malignant gynecologic Tumors. Clinical Pediatric Oncology. Sutow W.W., Fernbach, D.S., Vietti, T.J., The Mosby Co. Saint Louis 744, 1984. 
2. Julian P. Smith. M.D. and Felix Nutledge, M.D. Advances in chemotherapy for gynecologic eancer. Cancer 36: 669.1975.

3. James L.Breen, M.D., John F. Bonamo, M.D., and Wayne S. Maxsen, M.D.: Geniral tract tumors in children. Pediatr. Clin, North. Am. 28: 355, 1981.

4. Stevens, L.C.: The biology of teratomas inchding evidence indicating their origin from primordial germ cell, Ann. Biol. 1: 585, 1962.

5. Gellman, $J_{\text {.: }}$ The development of the gonads in man with consideration of the role of fetal endocrines and the histogenesis of ovarian tumor. Contrib. F.mbryol. 32: 83, 1948.

6. Slavton. K.E., Hreshchyshyn, M.M., Silverberg, S.V et al: Treatment of malignant ovarian germ cell tumors. Response to vincristine, dactomycin and ciglohospamide (preliminary report). Cancer 42: 390,1978 .

7. Gersbenson, P.A., Del Junco G., Herson, J., Putledge, F.M.: Endodermal sinus of the ovary: The M.D. Anderson experience. Obstet. Gynecol. 61: $194,1983$.
8. Kosholle, A.M., Favara, B.E., Hays, T., Mayor, F.S., Boiley, W.C.: Management of intrature teratoma of the ovary in clildren by conservative resection and chemo therapy. J. Pediatr. Surg. M.: 839, 1976.

9. Dara, P., Rich, W.M., Hodel, K., Disara, P.J.: Longterm discase-free surviral in inmature teratoma of the ovary Cancer 50: 159, 1982.

10. Lawrence H. Emhom, M.D. and john Donohue.: Cisdianmine dichloroplatinum, vinblastine, and bleonycin combination Chemotherapy in disseminated testicular Cancer. Ann. Intern, Med. 87: 293,1977

11. Moore, M.R., Garret, P.R., Walton, K.N. et al. Evaluation of humas chorionic gonadotropin and alplia Feto protein in benign and malignant testicular disorders. Surg. Gynecol. Obstetr. 117: 167, 1978.

12. Toshibico Ihara, Koso Ohama, Hideo Satoh, Tsumeo Fuji, Kazushi Nomuro and Atsushi Fijiwara: Histologic grade and karyotipe of immature teratoma of thic ovary. Cancer S4: 2988, 1984. 